\title{
La necesidad de una mirada reflexiva hacia los conflictos en Cochrane y la oportunidad de una salida superadora
}

The need for a reflexive look at the conflicts in Cochrane and the opportunity for a way out

Agustín Ciapponis

Ciapponi A. La necesidad de una mirada reflexiva hacia los conflictos en Cochrane y la oportunidad de una salida superadora. Evid Actual Pract Ambul. 2018;21(3):73-74.

Es muy difícil brindar una mirada objetiva a la cadena de eventos institucionales y mediáticos aparentemente desencadenados por la controversia científica tras la crítica a la revisión Cochrane de la vacuna contra el HPV $\mathrm{HP}^{1,2}$. En este número de la revista EVIDEN$\mathrm{CIA}$ nos hemos referido en extenso a la controversia científica, ofreciendo nuestra interpretación ${ }^{3}$. Habría preferido no tener que salir de lo estrictamente científico para escribir este comentario, que por supuesto no intentará cerrar el tema, pero si brindar precisiones sobre declaraciones negativas e injustas que afectan el prestigio y el honor de una institución, como Cochrane, que hace enormes contribuciones a la salud mundial y a la que pertenezco desde hace 17 años. Puedo dar fe que el debate científico es bienvenido y las diferencias de opinión entre las diferentes voces Cochrane no es inesperado ni novedoso. Sin embargo, la confianza pública puede verse minada, y la salud pública resentida, si el debate se lleva a cabo de manera inapropiada.

Durante un cuarto de siglo, los investigadores, médicos, pacientes y decisores, entre otros interesados, han estado utilizando las nuevas y rigurosas herramientas basadas en la evidencia para producir colectivamente revisiones sistemáticas sobre intervenciones y más recientemente, sobre pruebas diagnósticas. La Revisión Cochrane se ha convertido en un patrón de oro de la evidencia, y una de las fuentes de consulta obligada para la toma de decisiones relacionadas con la salud ${ }^{4}$.

Esta breve introducción solo tiene por objeto explicar que la organización en cuestión tiene una importancia tal que al menos merece prudencia antes de emitir juicios superficiales sobre lo ocurrido durante el pasado setiembre.

Durante el mes de septiembre de 2018 el organismo de gobierno de Cochrane expulsó a uno de sus miembros fundadores: Peter Gøtzsche. Las razones de la expulsión habrían sido una ruptura por parte de Gøtzsche de los códigos de conducta de la organización y la violación de su política de portavocía (Spokeperson policy), si bien dada la existencia de políticas de confidencialidad de la organización el proceso no fue públicamente deliberado.

Aunque el proceso ocurrió luego de la publicación de su crítica ${ }^{2}$ a la revisión Cochrane de la vacuna contra el $\mathrm{HPV}^{1}$, una mínima observación científica permite entender que esta secuencia no es suficiente para establecer causalidad. En la declaración de la Junta de Gobierno Cochrane ante la Asamblea General Anual del 17/7/2018, en el marco del Colloquium Cochrane de Edimburgo, se remarcó que la decisión nada tiene que ver con la libertad de expresión y que no se trata de un debate científico ni de intolerancia a la disidencia. De hecho, las dificultades datan desde hace 15 años y mucho antes de la publicación del artículo crítico en BMJ-EBM, se había iniciado un proceso interno por lo que la Junta consideró inconductas serias repetidas pero que no difundió por cuestiones legales de confidencialidad.

Gøtzsche podría ser clasificado como un purista de la medicina basada en la evidencia ${ }^{5}$. Descripto por una publicación como "un defensor audaz de la integridad en la medicina", es coautor de las declaraciones CONSORT, PRISMA, STROBE y SPIRIT sobre cómo emprender y publicar investigaciones ${ }^{6}$. Su controvertida posición respecto a que los expertos en contenidos clínicos pueden ser innecesarios en los equipos de revisión sistemática, lo describe bien. Mantiene una verdadera cruzada contra el ses- go en la investigación y contra las políticas basadas en lo que interpreta como evidencia sesgada o influenciada por la industria 7,8

Más allá de las razones que desencadenaron la mediática expulsión de Gøtzsche de Cochrane, de la que fue miembro fundador y cuya misión y valores comparte, es necesario reflexionar cómo una organización que pretende ser inclusiva dirime un conflicto grave con uno de sus miembros. Uno puede preguntarse si la jerarquía y el prestigio de la persona en cuestión debería darle inmunidad absoluta y la respuesta clara es que no. Por supuesto que hay límites que deben ejercerse en el marco de la igualdad ante la ley. Pero, por otro lado, teniendo en cuenta la magnitud del impacto de tal decisión, considero que debieron haberse agotado más instancias que las utilizadas y de llegarse a la última de ellas a través de un consenso significativo, explicar con tal claridad dichas razones.

Muchas veces los conflictos se malignizan por vedetismos o por dar muestras de autoridad, pero lo que debió haber primado es el rol clave de la organización a la que pertenecemos. Las estructuras válidas en sus inicios para albergar una pequeña red académica que se transformó en una organización internacional compleja y un gigante proyecto colaborativo global pueden ser ahora insuficientes. Actualmente se están actualizando y mejorando los protocolos internos de resolución de conflictos de manera imparcial y transparente, que incluyen instancias de apelación. Pero ¿qué hacer frente al presente conflicto?

Trisha Greenhalgh, profesora de Ciencias de la Salud de Atención Primaria en la Universidad de Oxford, no cree que Cochrane esté experimentando una crisis de moralidad o democracia y que debería bajarse un poco la intensidad mediática mientras se pone en orden la propia casa. Destaca, por otro lado, la marca, que como siempre, representa el rigor, la independencia y el compromiso de usar la ciencia para lograr cuidados de salud de alta calidad y justicia social ${ }^{5}$.

En la misma línea se encuentra la opinión de Ray Moynihan publicada en el blog del BMJ titulada "Paremos las llamas y la sangre en la Cochrane: hay demasiado en juego"s.

Para este prestigioso periodista de enormes contribuciones al entendimiento y difusión de la medicina basada a la evidencia y al resguardo de conflicto de intereses, las decenas de miles de personas de todo el mundo que crearon la Colaboración Cochrane y sus resúmenes de evidencia están contribuyendo a uno de los mayores esfuerzos científicos de la humanidad.

Lo que está en juego en la actual "lucha sangrienta" que está teniendo lugar en la Junta de Gobierno Cochrane no es solo la credibilidad de las personas a nivel individual o de las organizaciones, es el futuro de la evidencia confiable y robusta. Ironiza, que, si hubiera un fármaco o una terapia cognitivo conductual que redujese de manera significativa los síntomas de soberbia y promoviera la humildad, la recomendaría en dosis altas a las personas clave de este asunto. Confía en aprovechar este desafío como una oportunidad. La crisis más grande aquí es la amenaza a la credibilidad o confiabilidad de la evidencia en el sistema de salud y a la confianza pública erosionada por los perniciosos enredos financieros entre la industria y aquellas personas que evalúan y usan sus productos.

Quince años atrás, Cochrane decidió endurecer su política y rechazar firmemente la idea de que las compañías pudieran pa-

$\S$ Servicio de Medicina Familiar y Comunitaria del Hospital Italiano de Buenos Aires y director de Cochrane Argentina y del Centro Cochrane Instituto de Efectividad Clínica y Sanitaria. IECS. agustin.ciapponi@ hospitalitaliano.org.ar 
trocinar las revisiones Cochrane. Aun así, la política Cochrane, renovada en el 2014, permite que personas con conflictos de interés revisen la evidencia acerca de sus mismos productos, si constituyen una minoría en el equipo revisor. Moynihan aboga en su nota por un endurecimiento aún mayor "... dado lo que conocemos a día de hoy acerca del sesgo sistémico introducido en los estudios patrocinados por la industria y de la naturaleza atroz del comportamiento de la mayoría de sus departamentos de marketing (con los líderes de opinión en sus filas), se ha convertido en un anatema el que las personas con conflictos de interés revisen la evidencia relacionada con productos en la que están involucrados. Cochrane tiene la oportunidad de proveer liderazgo global limpiando este desorden, al igual que el BMJ tiene la intención de hacerlo con su nueva política de búsqueda de personas investigadoras sin conflictos de interés para la autoría de material educativo influyente..." estas estrategias por mejorar la confianza pública en la investigación médica diría también que es importante que las revisiones Cochrane muestren un mayor y explícito escrutinio de las controvertidas definiciones de enfermedad utilizadas por los investiga- dores, especialmente cuando el umbral de diagnóstico es inapropiadamente bajo y puede conducir a casos de sobrediagnóstico y sobretratamiento". Aclara que sus palabras no pretenden tomar partido sino contribuir a calmar, enfriar y estimular la búsqueda de una resolución al conflicto dado su excepcional interés en que esta colaboración global sobreviva y prospere.

En el marco de la red Cochrane Iberoamericana consideramos que expulsar a un miembro de una organización nunca puede convertirse ni parecer un proceso sumario, de cierre abrupto y sin la imprescindible transparencia. Sin duda, así es como nosotros y otras muchas personas de nuestra organización y ajenas a la misma hemos percibido el desenlace de este conflicto que ha dañado la imagen de la Colaboración. Por el contrario, quitarle urgencia y rever con transparencia absoluta el proceso sería probablemente la conducta institucional más apropiada tanto interna como externamente.

Deseo fuertemente que el sentido común prevalezca por sobre las disputas personales teniendo en mente la magnitud de lo que está en juego.

Declaración de conflicto de interés: Todas las opiniones aquí vertidas son a título estrictamente personal y de ninguna forma constituyen una posición oficial de Cochrane. Mi involucramiento de larga data en Cochrane hace que mi principal interés sea el que define la misión Cochrane: evidencia confiable, decisiones informadas, mejor salud.

\section{Referencias}

1. Arbyn M y col. Prophylactic vaccination against human papillomaviruses to prevent cervical cancer and its precursors. Cochrane Database of Systematic Reviews 2018 , Issue 5. Art. No.: CD009069. DOI: 10.1002/14651858.CD009069.pub3

2. Jørgensen $L$ y col. The Cochrane HPV vaccine review was incomplete and ignored important evidence of bias. BMJ evidence-based medicine, 2018 , p. bmjebm-2018-111012. 3. Ciapponi A y col. Vacunación contra el HPV para prevenir el cáncer de cuello uterino y sus precursores: una evidencia válida a pesar de las críticas. Evid Actual Pract Ambul 2018;21(3):xx-xx. Comentado de: Arbyn M y col. Prophylactic vaccination against human papillomaviruses to prevent cervical cancer and its precursors. Cochrane Database of Systematic Reviews 2018, Issue 5. Art. No.: CD009069. DOI: 10.1002/14651858.CD009069.pub3

4. Page M y col. Epidemiology and reporting characteristics of systematic reviews of biomedical research: a cross-sectional study. PLOS Medicine 2016;13(5):e1002028. https:// doi.org/10.1371/journal.pmed.1002028

5. Greenhalgh T: The Cochrane Collaboration—what crisis? Accedido el 7/10/18 desde: https://blogs.bmj.com/bmj/2018/09/17/trish-greenhalgh-the-cochrane-collaboration-whatcrisis/

6. Peter Gøtzsche receives 2016 HealthWatch Award. HealthWatch Newsletter. Accessed 15/9/18 on https://wwwhealthwatch-ukorg/images/Newsletters/Number_103_Highlightspdf 2016; Autumn:2.

7. Gøtzsche P. Mammography Screening: Truth, Lies and Controversy. London: CRC Press 2012

8. Gøtzsche P. Deadly Medicines and Organised Crime: How Big Pharma Has Corrupted Healthcare. London: CRC Press 2013.

9. Ray Moynihan: Let's stop the burning and the bleeding at Cochrane-there's too much at stake. Accedido el 7/10/18 desde: https://blogs.bmj.com/bmj/2018/09/17/ray-moynihan-lets-stop-the-burning-and-the-bleeding-at-cochrane-theres-too-much-at-stake/

10. Mabel Chew M y col. Medical journals and industry ties. BMJ 2014;349:g7197.

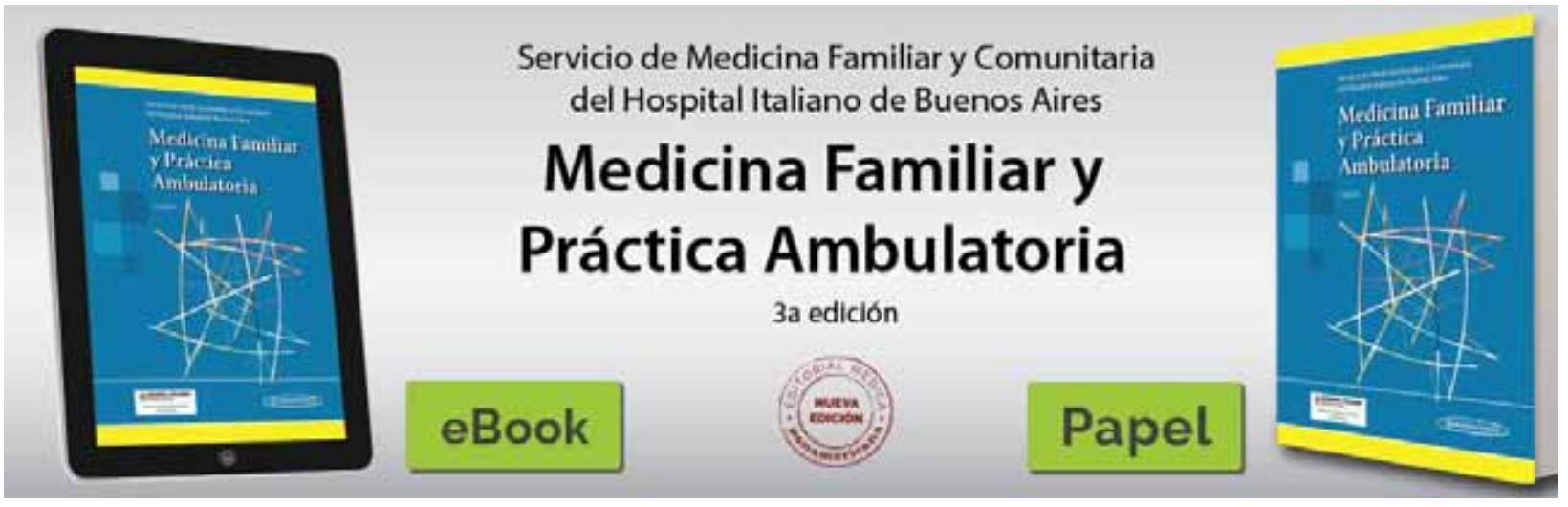

